\title{
The Role of the Inflammasome in Nonmyeloid Cells
}

\author{
Amir S. Yazdi • Stefan K. Drexler • Jürg Tschopp
}

Received: 30 May 2010 / Accepted: 1 June 2010 / Published online: 27 June 2010

(C) Springer Science+Business Media, LLC 2010

\begin{abstract}
Inflammasomes are cytosolic multiprotein complexes that can proteolytically activate caspase-1. Activated caspase- 1 is needed for the maturation and secretion of interleukin (IL)-1 $\beta$ and IL-18. In the past decade, there has been tremendous progress in our knowledge of inflammasome function and IL-1 signaling, mainly in cells of the innate immune system, such as monocytes, macrophages, neutrophils, and dendritic cells. Because nonimmune cells, including keratinocytes, synovial cells, or astrocytes, can form an interface between the body and the environment or a defined compartment (brain, joint), they are important guardians for the detection of danger signals and the consecutive initiation of an inflammatory response. They are present in anatomical compartments that are less accessible to myeloid cells and thus can fulfill tasks usually performed by residential macrophages. This review focuses on recent progress in our understanding of the processing and functional role of IL-1 in epithelial, mesenchymal, and neuronal cells and in conditions such as tissue repair.
\end{abstract}

Keywords Inflammasome $\cdot$ nonmyeloid cells $\cdot$ interleukin

\section{Introduction}

It has been 25 years since the cloning of Interleukin (IL)- $1 \beta$ and IL- $1 \alpha[1,2]$. Originally, these cytokines were described as fever-inducing agents, and it is now clear that they are

Yazdi and Drexler contributed equally to this work

A. S. Yazdi $\cdot$ S. K. Drexler · J. Tschopp $(\bowtie)$

Department of Biochemistry, University of Lausanne,

Chemin des Boveresses 155,

1066 Epalinges, Switzerland

e-mail: j.tschopp@unil.ch among the most potent proinflammatory cytokines expressed in mammals [3, 4]. While IL- $1 \beta$ and IL- $1 \alpha$ share similar physiological properties, their processing is distinct. Pathways leading to IL- $1 \alpha$ cleavage are yet to be characterized in detail, and the functional consequences of cleavage remain unclear as, unlike IL- $1 \beta$, IL- $1 \alpha$ does not have to be cleaved to exhibit biological activity. Initial studies described the $\mathrm{Ca}^{2+}$-dependent protease calpain as one protease able to process IL-1 $\alpha$ [5], although this has yet to be demonstrated genetically.

On the other hand, the mechanism of cleavage of pro-IL$1 \beta$ is well characterized. Eight years after the cloning of IL$1 \beta$, caspase-1 was identified as the essential protease that processes inactive pro-IL-1 $\beta$ to biologically active mature IL-1 $\beta$ [6]. Subsequently, the molecular pathways leading to caspase-1 activation were discovered. Large caspase-1 activating protein complexes termed the inflammasomes are formed in response to numerous biologically and chemically unrelated stimuli [7]. An inflammasome consists of three major components: (1) a receptor such as a nucleotide oligomerisation domain (NOD)-like receptor (NLR), which acts as an intracellular sensor and induces complex formation; (2) in most cases, the adaptor protein ASC (apoptosis speck-like protein containing a caspase recruitment domain (CARD)), which recruits pro-caspase-1 to the complex through homotypic CARD interactions; and, (3) pro-caspase-1 [7-9]. The close proximity of procaspase-1 in this complex results in autocatalytic cleavage of caspase- 1 and therefore its activation. To date, the NLR family consists of 22 human receptors that have been classified according to a common domain structure. While the function of most NLRPs remains elusive, NLRP1, NLRP3, and the NLRP-related protein, NLRC4 (IPAF), have been extensively characterized and are known to form inflammasomes that activate pro-caspase-1 [7, 10]. How- 
ever, these inflammasomes have primarily been studied in myeloid lineages, and their function in nonmyeloid cells and their role in noninflammatory processes are only beginning to emerge.

\section{Skin}

As skin is one of the body's largest primary barriers to the environment, one of its main functions is to protect the body against danger, such as chemical, physical, or microbial insults. The skin consists of two major compartments, the epidermis and the underlying dermis. Keratinocytes are the major cell type of the epidermis and they constantly renew from the basal layer to the differentiated corneal layer, which forms the main mechanical barrier that repels foreign agents and prevents dehydration. While innate immune cells such as macrophages and dermal dendritic cells are present in the dermis, Langerhans cells and epidermal dendritic cells are the only innate immune cells in the epidermal layer (Fig. 1a). Besides a small number of $\mathrm{T}$ cells (approximately $2 \%$ of the total skin), melanin pigment-producing melanocytes are the fourth major cell type in the epidermis [11].

To be able to sense pathogens and to elicit an immune response prior to infiltrating myeloid cells, keratinocytes can detect microbial or nonmicrobial danger signals through the recognition of conserved molecular patterns termed pathogen- or danger-associated molecular patterns
(PAMPs or DAMPs) (Fig. 1b). If presented to the outer membranes of the cell, these patterns can be sensed by Tolllike receptors, which are present either on the cell surface (TLR1, 2, 4, 5, 6) or on the endosomal membranes (TLR3, TLR9), to initiate immune responses $[12,13]$. NLRs are a recently identified group of proteins that are the cytosolic counterparts of TLRs. As mentioned above, some of the NLRs have the capacity to form multiprotein complexes termed inflammasomes, which leads to caspase-1 activation and the generation of mature biologically active IL-1 $\beta$. To date, the expression and function of the NLRP1 and the NLRP3 inflammasome, and of inflammatory caspases, have been demonstrated both in rodent and human skin [14]. The NLRP3 inflammasome, the most well-characterized inflammasome in myeloid cells, is known to be activated by ultraviolet B light, to induce sunburn-associated skin inflammation [15], and also in contact hypersensitivity reactions $[14,16]$, cutaneous $\mathrm{T}$ cell-mediated reactions directed against defined antigens. Watanabe et al. provided in vivo evidence for the dependency of contact hypersensitivity reactions on the presence of the IL-1 receptor (IL-1R) and the inflammasome components NLRP3 and ASC, while Sutterwala et al. further narrowed down the role of the inflammasome to the sensitization phase.

Caspase-1 and the inflammasome may not be only involved in cutaneous reactions directed against exogenous danger signals. Increased caspase-1 activity was detected in the lesional skin of psoriasis [17], which is a chronic, noninfectious inflammatory skin disease, characterized by
A)

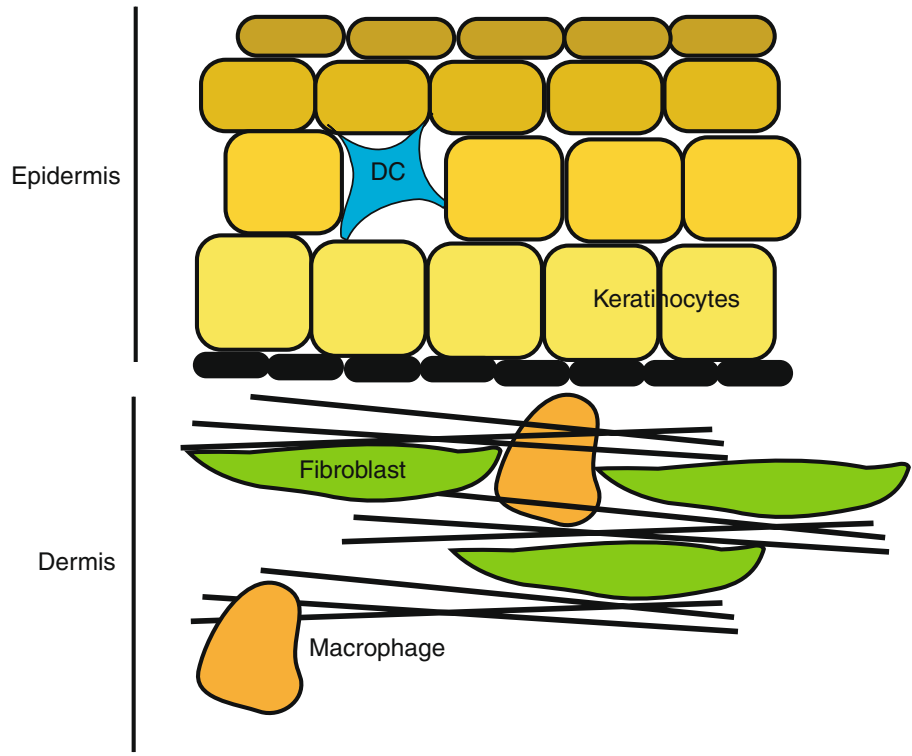

B)

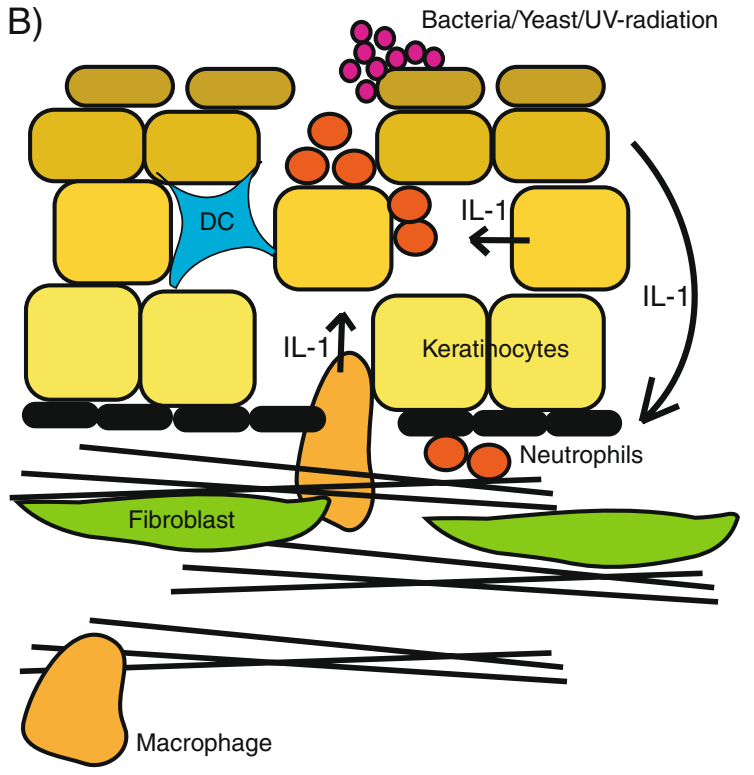

Fig. 1 Nonimmune cells are a source of proinflammatory cytokines, such as IL-1 $\beta$. Nonimmune cells, including keratinocytes, synovial cells, or astrocytes, can form an interface between the body and the environment or a defined compartment (brain, joint) that is not readily accessible to myeloid cells. Therefore, they are important guardians for the detection of danger signals and the consecutive initiation of an inflammatory response. By doing so, they are able to recruit myeloid cells, such as neutrophils and macrophages, to the site of insult 
silvery-white scaly patches or plaques. A second important autoinflammatory skin disease is vitiligo, which causes depigmented white patches due to the loss of melanocytes. In genetic association analyses, variants of NLRP1 were identified as a candidate gene for generalized vitiligo [18] or vitiligo-associated autoimmune diseases [19]. While these genetic association studies did not address the actual presence of NLRP1 in lesional skin, the protein expression of NLRP1 in rat skin was recently confirmed [20].

Human skin is known to possess caspase-1, caspase-5, ASC, pro-IL-1 $\alpha$, pro-IL-1 $\beta$, pIL-18 [15], NLRP1, and NLRP3 [14]. For murine skin, the expression pattern is controversial. On the one hand, Watanabe et al. exposed murine keratinocytes to the skin irritant 2,4,6-trinitrochlorobenzene (TNCB), leading to the secretion of mature IL- $1 \beta$ secretion, while others argue that $\mathrm{pIL}-1 \beta$ is absent in murine keratinocytes $[15,21]$.

\section{Connective Tissue and Tissue Repair}

The connective tissue supports and links other tissues of our body. Therefore, an intact connective tissue is necessary for the maintenance and remodeling of our body. Unlike other tissues, it has cells, namely, fibroblasts, scattered throughout the tissue, which maintain its integrity by producing extracellular matrix (ECM). Similar to the skin, an increasing amount of evidence suggests that fibroblasts, endothelial cells, and myoblasts can express IL- $1 \beta$ and process it in an inflammasome-dependent manner [22-25]. In this context, it seems that IL- $1 \beta$ is capable of influencing tissue repair and remodeling by inducing increased ECM production and regulating balance between cell proliferation and apoptosis.

Numerous publications have investigated the effects of IL-1 $\beta$ on vascular injury and myocardial infarction [26, 27]. It was found that the inhibition of IL-1R signaling led to a reduction of apoptosis in heart muscle tissue upon injury. This was achieved by overexpressing the naturally occurring IL-1R antagonist IL-1RA or through administration of IL-1RA [26, 27]. In both cases, the authors argue that inhibiting inflammation through IL-1RA leads to a reduction of cardiomyocyte apoptosis, thereby allowing accelerated recovery. Indeed, more recently, it was shown that primary murine myoblasts do express inflammasome components, such as NLRP3, ASC, and caspase-1, and are able to produce IL-1 $\beta$ [22]. Moreover, in a genetic model of myopathy, these inflammasome components were shown to be up-regulated. However, despite inflammasome expression, it is still unclear whether myoblasts process proIL-1 $\beta$ in an inflammasome-dependent manner.

The above studies suggest that inhibition of IL-1R signaling may allow a quickened recovery from myocardial infarction by preventing apoptosis and possibly by reducing fibrosis and scaring. Consistent with the latter hypothesis, it has been demonstrated that the IL-1R can mediate fibrotic tissue reactions in a murine model of lung injury. Gasse et al. [23] showed that bleomycin-induced lung injury leads to the production of IL-1 $\beta$, which is dependent on the inflammasome adaptor ASC. The resulting inflammation is caused by neutrophil infiltration, and, as a consequence of IL-1 $\beta$ production, increased tissue remodeling and fibrosis occur due to enhanced fibroblast proliferation and collagen deposition. More recently, the same group identified monosodium urate released from dying cells as one danger signal that can induce IL-1 $\beta$ in this model in an NLRP3-dependent manner [24]. However, it is still unclear whether the inflammatory infiltrate alone is responsible for IL-1 $\beta$ production or whether resident epithelial cells are also able to produce and process this cytokine.

It is likely that under conditions of tissue damage, IL- $1 \beta$ serves a dual function as a proinflammatory cytokine that attracts cells required for the disposal of dead cells as well as an inducer of tissue repair. One such condition is the inflamed joint of rheumatoid arthritis (RA) patients. IL-1 $\beta$ has been implicated in RA pathology based on data from human as well as murine models of the disease [28-30]. However, so far, it was mainly thought that IL- $1 \beta$ present in the joint is mainly produced by infiltrating myeloid cells. A study by Kolly et al. [25] characterized the expression of inflammasome components in human rheumatoid synovium. They identified NLRP3, ASC and caspase-1 not only in the myeloid cells infiltrating the joint but also in endothelial cells and synovial fibroblasts. Therefore, these cells might play an active role in the constant repair of the joint but also the pathology of RA.

\section{Brain}

The brain is the most sensitive organ of the body, having extremely limited regenerative potential. In resting, normal conditions, peripheral immune cells are unable to access the brain and the spinal cord, the central nervous system (CNS), due to the blood-brain barrier. The blood-brain barrier is formed by capillary endothelia and perivascular glial cells, and its permeability is altered by tumors or inflammatory conditions [31], enabling immune cells such as neutrophils or monocytes to enter the CNS under these conditions. The infiltrating immune cells are not the only source of inflammatory cytokines, as resident phagocytic cells such as microglia, the resident macrophages of the brain, or astrocytes ("macroglia"), large dendriform ectodermal cells that can connect both to neurons or to cerebral vessels, are able to synthesize a plethora of chemokines or cytokines [32]. It has been known for several years that 
microglia can produce IL-1 $\beta$ to promote tissue remodeling after CNS trauma [33], similar to the effect that IL-1 $\beta$ has in connective tissue.

Overactivation of caspase-1 due to gain of function mutations of NLRP3 can cause hereditary autoinflammatory diseases, such as Muckle-Wells syndrome and neonatal-onset multisystem inflammatory disease (NOMID, also known as chronic infantile neurological, cutaneous, articular (CINCA)) [34]. These disorders are clinically characterized by periodic fever accompanied by skin rashes, amyloid depositions, and, in the case of NOMID, severe neurological complications with hearing impairment and respond dramatically on the treatment with IL-1R antagonists. However, despite the obvious involvement of IL- $1 \beta$ on the CNS, the precise source of IL- $1 \beta$ in the brain in these rare disorders has not been identified.

Two of the most frequent neuronal diseases associated with increased levels of IL-1 $\beta$ are Alzheimer's disease (AD) and Huntington's disease (HD). In AD, amyloid $\beta$ can directly activate the NLRP3 inflammasome in microglia cells, leading to the secretion of mature IL-1 $\beta$ [38]. The amount of the proinflammatory cytokine is further increased as reactive astrocytes surrounding amyloid aggregates display an augmented IL-1 $\beta$ expression [35].

HD is an autosomal-dominant progressive neurodegenerative disorder associated with dementia and hyperkinetic attacks, most probably due to an imbalance of neuronal transmitters. Ona et al. [36] demonstrated increased caspase-1 activation mediated by an altered Huntingtin protein and treatment of the affected mice with the caspaseinhibitor zVAD-fmk slowed disease progression.

In opposition to $\mathrm{AD}$ and $\mathrm{HD}$, where IL-1 or caspase-1 appear to be required for disease progression, in a mouse model of Parkinson's disease, mice deficient in IL-1R1, the common receptor for both IL- $1 \alpha$ and IL-1 $\beta$, showed a worse disease outcome compared to the control cohort [37], implying IL-1 imparts a protective role in Parkinson's disease.

In accordance with skin, the functional role of IL-1 in human disease or murine disease models is better characterized than the actual tissue expression of NLRs. Murine microglia are known to harbor caspase-1, pro-IL-1 $\beta$, NLRP3, and caspase-1, similar to macrophages outside the CNS [38]. de Rivero Vaccari et al. [39] demonstrated the presence of caspase-1, caspase-11, ASC, and NLRP1 in motor neurons in the ventral horn of spinal cords, with increased expression upon spinal cord injury. While spinal cord injury induces processing of IL-1 $\beta$ and IL-18, with an accumulation of intracellular cleaved IL-1 $\beta$ and IL-18, whether these mature cytokines are efficiently secreted has not been demonstrated.

Yin et al. [40] determined the expression of NLRs, IL$1 \beta$, and caspase- 1 at the RNA level using various tissue
cDNA libraries, including the brain, and concluded from their expression levels that the brain could be classified as "ready to go" for NLRP1 and NLRP3 inflammasome activation. However, due to the utilization of a tissue cDNA library, the exact cell type expressing the above inflammasome components remains unclear. Moreover, it cannot be excluded that contaminating blood cells or the lack of a priming signal might bias the above analysis.

\section{Conclusions}

Historically, it has been assumed that the only sources of IL-1 $\beta$ are cells of myeloid origin infiltrating the site of inflammation. However, it is now becoming clear that in several tissues, nonmyeloid cells are able to produce and process IL-1 $\beta$. It is, therefore, reasonable to assume that the production of IL-1 $\beta$ by tissue resident cells serves as an early response to infections in immune deprived tissues, such as the brain, joint, or the epidermis of the skin, thereby attracting immune cells. Moreover, besides its role as a potent proinflammatory cytokine, increasing evidence suggests that IL-1 $\beta$ may also function in tissue remodeling and repair.

Acknowledgments This work was supported by the Deutsche Forschungsgemeinschaft (A.S.Y.) and the Apo-Sys Consortium of the European Union (S.K.D.). We thank Dr. James Vince for his valuable advice.

\section{References}

1. Auron PE, Webb AC, Rosenwasser LJ, et al. Nucleotide sequence of human monocyte interleukin 1 precursor cDNA. Proc Natl Acad Sci USA. 1984;81:7907-11.

2. Lomedico PT, Gubler U, Hellmann CP, et al. Cloning and expression of murine interleukin-1 cDNA in Escherichia coli. Nature. 1984;312:458-62.

3. Ogilvie AC, Hack CE, Wagstaff J, et al. IL-1 beta does not cause neutrophil degranulation but does lead to IL-6, IL-8, and nitrite/ nitrate release when used in patients with cancer. J Immunol. 1996;156:389-94.

4. Tewari A, Buhles Jr WC, Starnes Jr HF. Preliminary report: effects of interleukin-1 on platelet counts. Lancet. 1990;336:712-4.

5. Kavita U, Mizel SB. Differential sensitivity of interleukin-1 alpha and -beta precursor proteins to cleavage by calpain, a calciumdependent protease. J Biol Chem. 1995;270:27758-65.

6. Thornberry NA, Bull HG, Calaycay JR, et al. A novel heterodimeric cysteine protease is required for interleukin-1 beta processing in monocytes. Nature. 1992;356:768-74.

7. Martinon F, Mayor A, Tschopp J. The inflammasomes: guardians of the body. Annu Rev Immunol. 2009;27:229-65.

8. Dostert C, Meylan E, Tschopp J. Intracellular pattern-recognition receptors. Adv Drug Deliv Rev. 2008;60:830-40.

9. Yazdi AS, Guarda G, D'Ombrain MC, Drexler SK. Inflammatory caspases in innate immunity and inflammation. J Innate Immun. 2010;2:228-37. 
10. Meylan E, Tschopp J, Karin M. Intracellular pattern recognition receptors in the host response. Nature. 2006;442:39-44.

11. Bos JD, Zonneveld I, Das PK, Krieg SR, van der Loos CM, Kapsenberg ML. The skin immune system (SIS): distribution and immunophenotype of lymphocyte subpopulations in normal human skin. J Invest Dermatol. 1987;88:569-73.

12. Lebre MC, van der Aar AM, van Baarsen L, et al. Human keratinocytes express functional Toll-like receptor 3, 4, 5, and 9. J Invest Dermatol. 2007;127:331-41.

13. Miller LS, Modlin RL. Human keratinocyte Toll-like receptors promote distinct immune responses. J Invest Dermatol. 2007;127: 262-3.

14. Watanabe H, Gaide O, Petrilli V, et al. Activation of the IL-1betaprocessing inflammasome is involved in contact hypersensitivity. J Invest Dermatol. 2007;127:1956-63.

15. Feldmeyer L, Keller M, Niklaus G, Hohl D, Werner S, Beer HD. The inflammasome mediates UVB-induced activation and secretion of interleukin-1beta by keratinocytes. Curr Biol. 2007;17: $1140-5$.

16. Sutterwala FS, Ogura Y, Szczepanik M, et al. Critical role for NALP3/CIAS1/Cryopyrin in innate and adaptive immunity through its regulation of caspase-1. Immunity. 2006;24:317-27.

17. Johansen C, Moeller K, Kragballe K, Iversen L. The activity of caspase-1 is increased in lesional psoriatic epidermis. J Invest Dermatol. 2007;127:2857-64.

18. Jin Y, Birlea SA, Fain PR, Spritz RA. Genetic variations in NALP1 are associated with generalized vitiligo in a Romanian population. J Invest Dermatol. 2007;127:2558-62.

19. Jin Y, Mailloux CM, Gowan $\mathrm{K}$, et al. NALP1 in vitiligoassociated multiple autoimmune disease. N Engl J Med. 2007;356: $1216-25$.

20. Li WW, Guo TZ, Liang D, et al. The NALP1 inflammasome controls cytokine production and nociception in a rat fracture model of complex regional pain syndrome. Pain. 2009;147:277-86.

21. Keller M, Ruegg A, Werner S, Beer HD. Active caspase-1 is a regulator of unconventional protein secretion. Cell. 2008;132:818-31.

22. Rawat R, Cohen TV, Ampong B et al. Inflammasome upregulation and activation in dysferlin-deficient skeletal muscle. Am J Pathol. 2010;176(6):2891-900

23. Gasse P, Mary C, Guenon I, et al. IL-1R1/MyD88 signaling and the inflammasome are essential in pulmonary inflammation and fibrosis in mice. J Clin Invest. 2007;117:3786-99.

24. Gasse P, Riteau N, Charron S, et al. Uric acid is a danger signal activating NALP3 inflammasome in lung injury inflammation and fibrosis. Am J Respir Crit Care Med. 2009;179:903-13.

25. Kolly L, Karababa M, Joosten LA, et al. Inflammatory role of ASC in antigen-induced arthritis is independent of caspase-1, NALP-3, and IPAF. J Immunol. 2009;183:4003-12.

26. Abbate A, Salloum FN, Vecile E, et al. Anakinra, a recombinant human interleukin-1 receptor antagonist, inhibits apoptosis in experimental acute myocardial infarction. Circulation. 2008;117: 2670-83.

27. Suzuki K, Murtuza B, Smolenski RT, et al. Overexpression of interleukin-1 receptor antagonist provides cardioprotection against ischemia-reperfusion injury associated with reduction in apoptosis. Circulation. 2001;104:1308-13.

28. Drexler SK, Foxwell BM. The role of toll-like receptors in chronic inflammation. Int J Biochem Cell Biol. 2010;42:506-18.

29. Drexler SK, Kong P, Inglis J et al. SIGIRR/TIR8 is an inhibitor of TLR signalling in primary human cells and regulates inflammation in models of rheumatoid arthritis. Arthritis Rheum. 2010. doi:10.1002/art.27517.

30. Drexler SK, Kong PL, Wales J, Foxwell BM. Cell signalling in macrophages, the principal innate immune effector cells of rheumatoid arthritis. Arthritis Res Ther. 2008;10:216.

31. Lossinsky AS, Shivers RR. Structural pathways for macromolecular and cellular transport across the blood-brain barrier during inflammatory conditions. Histol Histopathol. 2004;19:53564. Review.

32. Miyake T, Hattori T, Fukuda M, Kitamura T, Fujita S. Quantitative studies on proliferative changes of reactive astrocytes in mouse cerebral cortex. Brain Res. 1988;451:133-8.

33. Herx LM, Rivest S, Yong VW. Central nervous system-initiated inflammation and neurotrophism in trauma: IL-1 beta is required for the production of ciliary neurotrophic factor. J Immunol. 2000;165:2232-9.

34. Neven B, Callebaut I, Prieur AM, et al. Molecular basis of the spectral expression of CIAS1 mutations associated with phagocytic cell-mediated autoinflammatory disorders CINCA/NOMID, MWS, and FCU. Blood. 2004;103:2809-15.

35. Apelt J, Schliebs R. Beta-amyloid-induced glial expression of both pro- and anti-inflammatory cytokines in cerebral cortex of aged transgenic Tg2576 mice with Alzheimer plaque pathology. Brain Res. 2001;894:21-30.

36. Ona VO, Li M, Vonsattel JP, et al. Inhibition of caspase-1 slows disease progression in a mouse model of Huntington's disease. Nature. 1999;399:263-7.

37. Parish CL, Finkelstein DI, Tripanichkul W, Satoskar AR, Drago J, Horne MK. The role of interleukin-1, interleukin-6, and glia in inducing growth of neuronal terminal arbors in mice. J Neurosci. 2002;22:8034-41.

38. Halle A, Hornung V, Petzold GC, et al. The NALP3 inflammasome is involved in the innate immune response to amyloid-beta. Nat Immunol. 2008;9:857-65.

39. de Rivero Vaccari JP, Lotocki G, Marcillo AE, Dietrich WD, Keane RW. A molecular platform in neurons regulates inflammation after spinal cord injury. J Neurosci. 2008;28:3404-14.

40. Yin Y, Yan Y, Jiang X, et al. Inflammasomes are differentially expressed in cardiovascular and other tissues. Int J Immunopathol Pharmacol. 2009;22:311-22. 